\title{
SEQUENCES IN THE MAXIMAL IDEAL SPACE OF $H^{\infty}$
}

\author{
SHELDON AXLER AND PAMELA GORKIN
}

(Communicated by Palle E. T. Jorgensen)

\begin{abstract}
This paper studies the behavior of sequences in the maximal ideal space of the algebra of bounded analytic functions on an arbitrary domain. The main result states that for any such sequence, either the sequence has an interpolating subsequence or infinitely many elements of the sequence lie in the same Gleason part.
\end{abstract}

\section{INTRODUCTION}

Fix a positive integer $N$ and fix a nonempty open subset $\Omega$ of $\mathbf{C}^{N}$. The Banach algebra of bounded analytic functions on $\Omega$ is denoted by $H^{\infty}(\Omega)$; its maximal ideal space is denoted by $M\left(H^{\infty}(\Omega)\right)$. This paper studies the behavior of sequences in $M\left(H^{\infty}(\Omega)\right)$.

The maximal ideal space $M\left(H^{\infty}(\Omega)\right)$ consists of the multiplicative linear functionals from $H^{\infty}(\Omega)$ onto the complex plane C. For $\varphi, \tau \in M\left(H^{\infty}(\Omega)\right)$, the pseudohyperbolic distance between $\varphi$ and $\tau$, denoted $d_{\Omega}(\varphi, \tau)$, is defined by

$$
d_{\Omega}(\varphi, \tau)=\sup \left\{|\tau(f)|: f \in H^{\infty}(\Omega),\|f\|_{\infty}<1 \text {, and } \varphi(f)=0\right\}
$$

here $\|f\|_{\infty}$ is the usual supremum norm defined by

$$
\|f\|_{\infty}=\sup \{|f(z)|: z \in \Omega\} .
$$

As is well known, $d_{\Omega}$ is a metric on $M\left(H^{\infty}(\Omega)\right)$ with the property that any two open balls of radius 1 are either equal or disjoint (see, for example, Sections 1 and 2 of [1]). The open balls of radius 1 are called the Gleason parts of $M\left(H^{\infty}(\Omega)\right)$. Specifically, if $\varphi \in M\left(H^{\infty}(\Omega)\right)$, then the Gleason part of $\varphi$, denoted $G(\varphi)$, is defined by

$$
G(\varphi)=\left\{\tau \in M\left(H^{\infty}(\Omega)\right): d_{\Omega}(\varphi, \tau)<1\right\} .
$$

A sequence $\left(\varphi_{n}\right)_{n=1}^{\infty} \subset M\left(H^{\infty}(\Omega)\right)$ is called an interpolating sequence if for every bounded sequence of complex numbers $\left(\lambda_{n}\right)_{n=1}^{\infty}$, there exists $f \in H^{\infty}(\Omega)$ such that

$$
\varphi_{n}(f)=\lambda_{n} \quad \text { for every } n \in \mathbf{Z}^{+} \text {; }
$$

here $\mathbf{Z}^{+}$denotes the set of positive integers.

Received by the editors March 13, 1989.

1980 Mathematics Subject Classification (1985 Revision). Primary 46J15; Secondary 30H05.

Key words and phrases. Maximal ideal space, Gleason parts, interpolating sequences.

Both authors were partially supported by the National Science Foundation. 
Although $d_{\Omega}$ is a metric on $M\left(H^{\infty}(\Omega)\right)$, whenever we use topological notions (closure, limits, etc.) on $M\left(H^{\infty}(\Omega)\right)$, unless explicitly stated otherwise we are referring to the weak- ${ }^{*}$ topology that $M\left(H^{\infty}(\Omega)\right)$ inherits as a subset of the dual of $H^{\infty}(\Omega)$. With this topology, $M\left(H^{\infty}(\Omega)\right)$ becomes a compact Hausdorff space. Except in some trivial cases (for example, when $H^{\infty}(\Omega)$ consists of only the constant functions), the topology on $M\left(H^{\infty}(\Omega)\right)$ is not equal to the topology induced by the metric $d_{\Omega}$.

The next section contains the main result of this paper, Theorem 3, which states that if $\left(\varphi_{n}\right)_{n=1}^{\infty}$ is a sequence in $M\left(H^{\infty}(\Omega)\right)$ and $\varphi$ is a limit point of this sequence, then either $\varphi_{n}$ is contained in the Gleason part of $\varphi$ for infinitely many $n$ or $\left(\varphi_{n}\right)_{n=1}^{\infty}$ has an interpolating subsequence. As corollaries, we show (Corollary 9) that if $\varphi_{n} \rightarrow \varphi$, then $\varphi_{n}$ is in the Gleason part of $\varphi$ for all but finitely many $n$ and (Corollary 11) that if $\left(\varphi_{n}\right)_{n=1}^{\infty}$ is a sequence in $M\left(H^{\infty}(\Omega)\right)$ such that each $\varphi_{n}$ lies in a distinct Gleason part, then $\left(\varphi_{n}\right)_{n=1}^{\infty}$ has an interpolating subsequence. These statements would not be true if sequences were replaced by nets.

The final section of the paper specializes to the case of the open unit disk $D$ in the complex plane. Corollary 12 states that the path connected components of $M\left(H^{\infty}(D)\right)$ are precisely the Gleason parts. Corollary 13 shows that although the topology induced by the metric $d_{D}$ is not equal to the usual topology on $M\left(H^{\infty}(D)\right)$, the two topologies have the same convergent sequences.

We thank Hans-Martin Lingenberg for calling our attention to reference [2].

\section{DomaINS IN $\mathbf{C}^{N}$}

To prove our main result (Theorem 3), we will need two lemmas. For our first lemma, we need to know a bit about the pseudohyperbolic metric on the open unit disk $D$.

We can think of $D$ as a subset of $M\left(H^{\infty}(D)\right)$ by identifying each point of $D$ with the multiplicative linear functional of point evaluation at that point. With this identification, an easy computation using the Schwarz Lemma shows that

$$
d_{D}(w, z)=\left|\frac{w-z}{1-\bar{w} z}\right| \quad \text { for all } w, z \in D .
$$

Given $w_{1}, w_{2}, z_{1}, z_{2} \in D$ with $d_{D}\left(w_{1}, w_{2}\right)=d_{D}\left(z_{1}, z_{2}\right)$, the explicit description of the analytic automorphisms of $D$ (one-to-one analytic maps of $D$ onto $D$ ) shows that there exists an analytic automorphism $b$ of $D$ such that $b\left(w_{1}\right)=z_{1}$ and $b\left(w_{2}\right)=z_{2}$.

The idea for the statement and proof of the following lemma comes from [5], Chapter VII, Theorem 2.2.

Lemma 1. Let $\varphi, \tau \in M\left(H^{\infty}(\Omega)\right)$ be such that $\tau \notin G(\varphi)$. Let $\varepsilon>0$. Then there exist $f, g \in H^{\infty}(\Omega)$ such that

$$
\begin{array}{ll}
\varphi(f)=0, & \tau(f)=1, \\
\varphi(g)=1, & \tau(g)=0,
\end{array}
$$


and

$$
|f(z)|+|g(z)|<1+\varepsilon \quad \text { for every } z \in \Omega \text {. }
$$

Proof. Let

$$
\delta=1-1 / \sqrt{1+2 \varepsilon}
$$

and let $t=d_{D}(1-\delta,-1+\delta)$. Because $d_{\Omega}(\varphi, \tau)=1$, there exists $s \in H^{\infty}(\Omega)$ such that $\|s\|_{\infty}<1$ and

$$
\varphi(s)=0 \quad \text { and } \quad \tau(s)=t .
$$

Clearly $d_{D}(0, t)=d_{D}(1-\delta,-1+\delta)$, so there is an analytic automorphism $b$ of $D$ such that

$$
b(0)=1-\delta \quad \text { and } \quad b(t)=-1+\delta .
$$

Let

$$
h=\frac{b \circ s}{1-\delta}
$$

Then

$$
\|h\|_{\infty}<\sqrt{1+2 \varepsilon} \quad \text { and } \quad \varphi(h)=1, \tau(h)=-1
$$

where the equations $\varphi(h)=1, \tau(h)=-1$ follow from our conditions on $b$ and $s$ and Lemma 6.3 of [7]. Now let

$$
f=\left(\frac{1-h}{2}\right)^{2} \quad \text { and } \quad g=\left(\frac{1+h}{2}\right)^{2} \text {. }
$$

Then $f$ and $g$ are in $H^{\infty}(\Omega)$ and

$$
\begin{array}{lll}
\varphi(f)=0, & \tau(f)=1, \\
\varphi(g)=1, & \tau(g)=0 .
\end{array}
$$

Furthermore, if $z \in \Omega$, then

$$
\begin{aligned}
|f(z)|+|g(z)| & =\frac{(1-h(z))(1-\bar{h}(z))+(1+h(z))(1+\bar{h}(z))}{4} \\
& =\frac{1+|h(z)|^{2}}{2}<1+\varepsilon,
\end{aligned}
$$

completing the proof.

The following lemma will be used in the proof of Theorem 3 .

Lemma 2. Let $j$ be an integer greater than 1 , and let $a_{1}, \ldots, a_{j}, b_{1}, \ldots, b_{j}$ be nonnegative numbers. Let $\varepsilon_{1}, \ldots, \varepsilon_{j}$ be nonnegative numbers such that

$$
a_{k}+b_{k}<1+\varepsilon_{k} \quad \text { for } k=1, \ldots, j .
$$

Then

$$
a_{1}+a_{2} b_{1}+a_{3} b_{1} b_{2}+\cdots+a_{j} b_{1} b_{2} \cdots b_{j-1}<\left(1+\varepsilon_{1}\right)\left(1+\varepsilon_{2}\right) \cdots\left(1+\varepsilon_{j}\right) .
$$


Proof. We will prove this lemma by induction. For $j=2$, we have

$$
\begin{aligned}
a_{1}+a_{2} b_{1} & \leq a_{1}+b_{1}\left(a_{2}+b_{2}\right) \\
& \leq a_{1}+b_{1}\left(1+\varepsilon_{2}\right) \\
& \leq a_{1}\left(1+\varepsilon_{2}\right)+b_{1}\left(1+\varepsilon_{2}\right) \\
& <\left(1+\varepsilon_{1}\right)\left(1+\varepsilon_{2}\right),
\end{aligned}
$$

as desired.

Now suppose that the lemma holds for $j-1$. Then

$$
\begin{aligned}
a_{1}+a_{2} b_{1}+a_{3} b_{1} b_{2} & +\cdots+a_{j} b_{1} \cdots b_{j-1} \\
& =a_{1}+b_{1}\left(a_{2}+a_{3} b_{2}+a_{4} b_{2} b_{3}+\cdots+a_{j} b_{2} \cdots b_{j-1}\right) \\
& \leq a_{1}+b_{1}\left(1+\varepsilon_{2}\right)\left(1+\varepsilon_{3}\right) \ldots\left(1+\varepsilon_{j}\right) \\
& \leq a_{1}\left(1+\varepsilon_{2}\right)\left(1+\varepsilon_{3}\right) \ldots\left(1+\varepsilon_{j}\right)+b_{1}\left(1+\varepsilon_{2}\right)\left(1+\varepsilon_{3}\right) \ldots\left(1+\varepsilon_{j}\right) \\
& <\left(1+\varepsilon_{1}\right)\left(1+\varepsilon_{2}\right) \ldots\left(1+\varepsilon_{j}\right),
\end{aligned}
$$

and so the lemma holds for $j$, completing the proof.

We can now prove Theorem 3, the main result of the paper. Note that by the nested intersection property for compact sets, for every sequence $\left(\varphi_{n}\right)_{n=1}^{\infty}$ in $M\left(H^{\infty}(\Omega)\right)$, there exists $\varphi \in \bigcap_{n=1}^{\infty} \overline{\left\{\varphi_{n}, \varphi_{n+1}, \ldots\right\}}$.

Theorem 3. Let $\left(\varphi_{n}\right)_{n=1}^{\infty}$ be a sequence in $M\left(H^{\infty}(\Omega)\right)$, and let

$$
\varphi \in \bigcap_{n=1}^{\infty} \overline{\left\{\varphi_{n}, \varphi_{n+1}, \ldots\right\}} .
$$

Then either

$$
\varphi_{n} \in G(\varphi) \text { for infinitely many } n
$$

or

$$
\left(\varphi_{n}\right)_{n=1}^{\infty} \text { has an interpolating subsequence. }
$$

Proof. Suppose that there are only finitely many $n$ such that $\varphi_{n} \in G(\varphi)$. We will complete the proof by showing that in this case $\left(\varphi_{n}\right)_{n=1}^{\infty}$ has an interpolating subsequence.

Let $\left(\varepsilon_{n}\right)_{n=1}^{\infty}$ be a sequence of positive numbers such that

$$
\prod_{n=1}^{\infty}\left(1+\varepsilon_{n}\right)<2
$$

Because $\varphi_{n} \in G(\varphi)$ for only finitely many $n$, we can choose a positive integer $n_{1}$ such that

$$
\varphi_{k} \notin G(\varphi) \quad \text { for all } k \geq n_{1} .
$$

By Lemma 1, there exist $f_{1}, g_{1} \in H^{\infty}(\Omega)$ such that

$$
\begin{array}{ll}
\varphi\left(f_{1}\right)=0, & \varphi_{n_{1}}\left(f_{1}\right)=1, \\
\varphi\left(g_{1}\right)=1, & \varphi_{n_{1}}\left(g_{1}\right)=0,
\end{array}
$$


and

$$
\left|f_{1}(z)\right|+\left|g_{1}(z)\right|<1+\varepsilon_{1} \quad \text { for every } z \in \Omega .
$$

Now suppose that $j$ is an integer greater than 1 and that $f_{1}, \ldots, f_{j-1}, g_{1}$, $\ldots, g_{j-1}$, and $n_{1}, \ldots, n_{j-1}$ have been chosen such that

$$
\varphi\left(f_{1}\right)=\cdots=\varphi\left(f_{j-1}\right)=0 \quad \text { and } \quad \varphi\left(g_{1}\right)=\cdots=\varphi\left(g_{j-1}\right)=1 .
$$

Because

$$
\begin{gathered}
\left\{\psi \in M\left(H^{\infty}(\Omega)\right):\left|\psi\left(f_{1}\right)\right|+\left|\psi\left(f_{2} g_{1}\right)\right|+\cdots+\left|\psi\left(f_{j-1} g_{1} \ldots g_{j-2}\right)\right|<\frac{1}{4}\right. \text { and } \\
\left.\left|1-\psi\left(g_{1} \ldots g_{j-1}\right)\right|<\frac{1}{4}\right\}
\end{gathered}
$$

is an open subset of $M\left(H^{\infty}(\Omega)\right)$ containing $\varphi$, and because $\varphi$ is contained in the closure of $\left\{\varphi_{k}: k>n_{j-1}\right\}$, we can choose an integer $n_{j}>n_{j-1}$ such that

$$
\left|\varphi_{n_{j}}\left(f_{1}\right)\right|+\left|\varphi_{n_{j}}\left(f_{2} g_{1}\right)\right|+\cdots+\left|\varphi_{n_{j}}\left(f_{j-1} g_{1} \ldots g_{j-2}\right)\right|<\frac{1}{4}
$$

and

$$
\left|1-\varphi_{n_{j}}\left(g_{1} \ldots g_{j-1}\right)\right|<\frac{1}{4} \text {. }
$$

By Lemma 1, there exist $f_{j}, g_{j} \in H^{\infty}(\Omega)$ such that

$$
\begin{array}{ll}
\varphi\left(f_{j}\right)=0, & \varphi_{n_{j}}\left(f_{j}\right)=1, \\
\varphi\left(g_{j}\right)=1, & \varphi_{n_{j}}\left(g_{j}\right)=0,
\end{array}
$$

and

$$
\left|f_{j}(z)\right|+\left|g_{j}(z)\right|<1+\varepsilon_{j} \quad \text { for every } z \in \Omega .
$$

We now show that $\left(\varphi_{n_{j}}\right)_{j=1}^{\infty}$ is an interpolating sequence. Let $\ell^{\infty}$ denote the usual space of bounded sequences $\lambda=\left(\lambda_{n}\right)_{n=1}^{\infty}$ of complex numbers with norm defined by

$$
\|\lambda\|_{\infty}=\sup \left\{\left|\lambda_{n}\right|: n \in \mathbf{Z}^{+}\right\} \text {. }
$$

Let $\lambda \in \ell^{\infty}$ be such that $\|\lambda\|_{\infty}<1$. Define a function $h$ on $\Omega$ by

$$
h(z)=\lambda_{1} f_{1}(z)+\lambda_{2} f_{2}(z) g_{1}(z)+\cdots+\lambda_{j} f_{j}(z) g_{1}(z) \cdots g_{j-1}(z)+\cdots ;
$$

by Lemma 2 and inequalities (4) and (7), the partial sums of the absolute values of the above infinite series are all less than 2 , and so the infinite sum converges (pointwise on $\Omega$ ) and $\|h\|_{\infty}<2$. Because the pointwise limit of a sequence of uniformly bounded analytic functions is analytic, we conclude that $h \in H^{\infty}(\Omega)$.

Let $j \in \mathbf{Z}^{+}$. Then

$$
\begin{aligned}
\left|\varphi_{n_{j}}(h)-\lambda_{j}\right| \leq & \left|\varphi_{n_{j}}\left(\lambda_{1} f_{1}+\lambda_{2} f_{2} g_{1}+\cdots+\lambda_{j-1} f_{j-1} g_{1} \ldots g_{j-2}\right)\right| \\
& +\left|\lambda_{j}-\varphi_{n_{j}}\left(\lambda_{j} f_{j} g_{1} \ldots g_{j-1}\right)\right| \\
& +\mid \varphi_{n_{j}}\left(g _ { 1 } \ldots g _ { j } \left[\lambda_{j+1} f_{j+1}+\lambda_{j+2} f_{j+2} g_{j+1}\right.\right. \\
& \left.\left.+\mid \lambda_{j+3} f_{j+3} g_{j+1} g_{j+2}+\cdots\right]\right) \mid
\end{aligned}
$$


where the infinite sum

$$
\lambda_{j+1} f_{j+1}+\lambda_{j+2} f_{j+2} g_{j+1}+\lambda_{j+3} f_{j+3} g_{j+1} g_{j+2}+\ldots
$$

converges pointwise boundedly to some function in $H^{\infty}(\Omega)$ by Lemma 2 and inequalities (4) and (7). By inequality (5), the first term on the right-hand side of inequality (8) is less than $\frac{1}{4}$; by inequality (6), the second term on the righthand side of inequality ( 8$)$ is less than $\frac{1}{4}$; because $\varphi_{n_{j}}\left(g_{j}\right)=0$, the third term on the right-hand side of inequality (8) equals 0 . Thus we have shown that if $\lambda \in \ell^{\infty}$ with $\|\lambda\|_{\infty}<1$, then there exists $h \in H^{\infty}(\Omega)$ with $\|h\|_{\infty}<2$ such that

$$
\left|\varphi_{n_{j}}(h)-\lambda_{j}\right|<\frac{1}{2} \quad \text { for every } j \in \mathbf{Z}^{+} \text {. }
$$

In other words, using only functions from the ball of radius 2 of $H^{\infty}(\Omega)$, the sequence $\left(\varphi_{n_{j}}\right)_{j=1}^{\infty}$ can interpolate any element of the unit ball of $\ell^{\infty}$ with an error of at most $\frac{1}{2}$. This implies (see Lemma 4.1 of [4]) that $\left(\varphi_{n_{j}}\right)_{j=1}^{\infty}$ is an interpolating sequence, completing the proof.

On page 165 of [6], Kenneth Hoffman proved that if $\left(\varphi_{n}\right)_{n=1}^{\infty}$ is a convergent sequence in $M\left(H^{\infty}(D)\right) \backslash D$, then all but a finite number of the $\varphi_{n}$ lie in the same fiber (the fiber over a point $\lambda \in \partial D$ is the set of all $\varphi \in M\left(H^{\infty}(D)\right.$ ) such that $\varphi(z)=\lambda)$. The fibers are much bigger than the Gleason parts in $M\left(H^{\infty}(D)\right) \backslash D$, so the following corollary strengthens Hoffman's result (and applies to an arbitrary open subset $\Omega$ of $\mathbf{C}^{n}$ ). Note that the following corollary does not hold for nets, thus indicating the special role played by sequences.

Corollary 9. Let $\varphi \in M\left(H^{\infty}(\Omega)\right)$ and suppose that $\left(\varphi_{n}\right)_{n=1}^{\infty}$ is a sequence in $M\left(H^{\infty}(\Omega)\right)$ such that $\varphi_{n} \rightarrow \varphi$. Then

$$
\limsup _{n \rightarrow \infty} d_{\Omega}\left(\varphi, \varphi_{n}\right)<1 .
$$

In particular, $\varphi_{n} \in G(\varphi)$ for all sufficiently large $n$.

Proof. Suppose false, so that

$$
\limsup _{n \rightarrow \infty} d_{\Omega}\left(\varphi, \varphi_{n}\right)=1 .
$$

We began the proof of Theorem 3 by assuming that $\varphi_{n} \notin G(\varphi)$ for all sufficiently large $n$, and concluded that $\left(\varphi_{n}\right)_{n=1}^{\infty}$ has an interpolating subsequence. The assumption that $\varphi_{n} \notin G(\varphi)$ was only used so that Lemma 1 could be applied. Note, however, that the proof of Lemma 1 does not really require that $\tau \notin G(\varphi)$, but only that $d(\varphi, \tau)$ be sufficiently close to 1 (how close depends upon the $\varepsilon$ of Lemma 1). Thus given the sequence $\left(\varepsilon_{n}\right)_{n=1}^{\infty}$ of the proof of Theorem 3, we can choose a subsequence of $d_{\Omega}\left(\varphi, \varphi_{n}\right)$ tending to 1 sufficiently rapidly so that the proof of Theorem 3 works (because $\varphi_{n} \rightarrow \varphi$, any subsequence of $\left(\varphi_{n}\right)_{n=1}^{\infty}$ captures $\varphi$ in its closure, so the hypothesis of Theorem 3 holds). In other words, $\left(\varphi_{n}\right)_{n=1}^{\infty}$ has an interpolating subsequence. However, no convergent sequence can be an interpolating sequence (because the sequence of 
alternating 1's and -1's cannot be interpolated). This contradiction completes the proof.

If $\Omega$ is connected, then the Shilov Idempotent Theorem ([3], Chapter III, Corollary 6.5) shows that $M\left(H^{\infty}(\Omega)\right)$ is connected. However, except for trivial cases (for example, when $H^{\infty}(\Omega)$ consists of only the constant functions), $M\left(H^{\infty}(\Omega)\right)$ is not path connected. The following corollary shows that each path connected component of $M\left(H^{\infty}(\Omega)\right)$ is contained in a Gleason part. If $\Omega$ is the unit disk, then the path connected components of $M\left(H^{\infty}(\Omega)\right)$ are precisely equal to the Gleason parts (see Corollary 12 in the next section). We do not know whether this remains true when $\Omega$ is a ball or polydisk in $\mathbf{C}^{N}(N>1)$. Theorem 5.5 of [4] (also see Theorem 3.3 and Corollary 6.4 of [4]) shows that there is a domain $\Omega$ in $\mathbf{C}$ such that $M\left(H^{\infty}(\Omega)\right)$ has a Gleason part that is not path connected.

Corollary 10. Each path connected component of $M\left(H^{\infty}(\Omega)\right)$ is contained in a Gleason part.

Proof. Let $\gamma:[0,1] \rightarrow M\left(H^{\infty}(\Omega)\right)$ be a continuous function. We need to prove that $\gamma(1) \in G(\gamma(0))$. Suppose that $\gamma(1) \notin G(\gamma(0))$. Let

$$
T=\sup \{t \in[0,1]: \gamma(t) \in G(\gamma(0))\}
$$

We now break the proof into two cases.

Case 1. Suppose that $\gamma(T) \in G(\gamma(0))$. Then $T<1$ and $\gamma(T+1 / n) \rightarrow \gamma(T)$, but for each $n$ we have $\gamma(T+1 / n) \notin G(\gamma(T))$, contradicting Corollary 9 .

Case 2. Suppose that $\gamma(T) \notin G(\gamma(0))$. Then $T>0$ and there exists a sequence $\left(t_{n}\right)_{n=1}^{\infty} \subset[0, T)$ such that $t_{n} \rightarrow T$ as $n \rightarrow \infty$ and $\gamma\left(t_{n}\right) \in G(\gamma(0))$ for every $n$. Thus $\gamma\left(t_{n}\right) \rightarrow \gamma(T) \notin G(\gamma(0))$, contradicting Corollary 9 and completing the proof.

The following corollary shows that except for trivial cases, $M\left(H^{\infty}(\Omega)\right)$ is rich in interpolating sequences.

Corollary 11. Let $\left(\varphi_{n}\right)_{n=1}^{\infty}$ be a sequence in $M\left(H^{\infty}(\Omega)\right)$ such that for distinct $m$ and $n, \varphi_{m}$ and $\varphi_{n}$ are contained in distinct Gleason parts. Then $\left(\varphi_{n}\right)_{n=1}^{\infty}$ has an interpolating subsequence.

Proof. By the nested intersection property, there exists $\varphi \in M\left(H^{\infty}(\Omega)\right)$ such that

$$
\varphi \in \bigcap_{n=1}^{\infty} \overline{\left\{\varphi_{n}, \varphi_{n+1}, \ldots,\right\}} .
$$

Our hypothesis implies that $\varphi_{n} \in G(\varphi)$ for at most one $n$. Thus by Theorem $3,\left(\varphi_{n}\right)_{n=1}^{\infty}$ has an interpolating subsequence, completing the proof. 


\section{THE UNIT DISK}

In the case where $\Omega$ is the unit disk in the complex plane, most of the results in the previous section were proved by A. Dufresnoy [2]. Dufresnoy's proofs require that each element of $M\left(H^{\infty}(\Omega)\right)$ have a unique representing measure on the Shilov boundary of $H^{\infty}(\Omega)$, and thus his proofs do not work even for nice domains such as balls or polydisks in $\mathbf{C}^{N}(N>1)$. Even in the case where $\Omega$ is the unit disk in $\mathbf{C}$, the proofs given in the last section (which apply to arbitrary domains in $\mathbf{C}^{N}$ ) seem to be easier than the proofs given by Dufresnoy.

Recall that $D$ denotes the open unit disk in the complex plane. For each $\varphi \in M\left(H^{\infty}(D)\right)$, there is a canonical map $L_{\varphi}$ of $D$ onto $G(\varphi)$; detailed information about these maps was discovered by Hoffman [7] (also see [5], Chapter X). In this section we use these canonical maps and Theorem 3 to prove two results about $M\left(H^{\infty}(D)\right)$.

We remarked before Corollary 10 that the following corollary does not hold for arbitrary domains.

Corollary 12. The path connected components of $M\left(H^{\infty}(D)\right)$ are precisely the Gleason parts.

Proof. Let $\varphi \in M\left(H^{\infty}(D)\right)$. Then $L_{\varphi}$ is a continuous map of $D$ onto $G(\varphi)$. Thus $G(\varphi)$ is path connected. By Corollary $10, G(\varphi)$ is not contained in a larger path connected subset of $M\left(H^{\infty}(D)\right)$. Thus $G(\varphi)$ is the path connected component of $M\left(H^{\infty}(D)\right)$ containing $\varphi$, completing the proof.

For $\psi$ a linear functional on $H^{\infty}(D)$, let $\|\psi\|$ denote the usual norm defined by

$$
\|\psi\|=\sup \left\{|\psi(f)|: f \in H^{\infty}(D) \text { and }\|f\|_{\infty}<1\right\} .
$$

The next corollary states that for a sequence in $M\left(H^{\infty}(D)\right)$, convergence in the usual weak- ${ }^{*}$ topology of $M\left(H^{\infty}(D)\right)$ is equivalent to norm convergence and to convergence in the metric $d_{D}$ (this is false if sequences are replaced by nets). We believe that Corollary 13 might remain true if the unit disk is replaced by an arbitrary domain in $\mathbf{C}^{N}$, but the tools used in the proof below are not available even for nice domains such as balls or polydisks in $\mathbf{C}^{N}(N>1)$.

The proof of the next corollary will use the following results (see [7] or Chapter $\mathrm{X}$ of [5]): For each $\varphi \in M\left(H^{\infty}(D)\right)$, there exists a continuous function $L_{\varphi}: D \rightarrow M\left(H^{\infty}(D)\right)$ such that

$$
\begin{gathered}
L_{\varphi}(D)=G(\varphi) \\
L_{\varphi}(0)=\varphi \\
\text { if } G(\varphi) \neq\{\varphi\}, \text { then } L_{\varphi} \text { is injective }
\end{gathered}
$$

if $f \in H^{\infty}(D)$, then the map that sends $w$ to $\left(L_{\varphi}(w)\right)(f)$ is analytic on $D$. The map from $D$ to $\mathbf{C}$ that sends $w$ to $\left(L_{\varphi}(w)\right)(f)$ will be denoted 
by $f \circ L_{\varphi}$; this notation is natural if $f$ is identified with its Gelfand transform. Thus if $f \in H^{\infty}(D)$ and $\varphi \in M\left(H^{\infty}(D)\right)$, then $f \circ L_{\varphi} \in H^{\infty}(D)$ and $\left\|f \circ L_{\varphi}\right\|_{\infty} \leq\|f\|_{\infty}$.

Corollary 13. Let $\left(\varphi_{n}\right)_{n=1}^{\infty}$ be a sequence in $M\left(H^{\infty}(D)\right)$ and let $\varphi \in$ $M\left(H^{\infty}(D)\right)$. Then the following are equivalent:

(a) $\varphi_{n} \rightarrow \varphi$ in $M\left(H^{\infty}(D)\right)$;

(b) $d_{D}\left(\varphi_{n}, \varphi\right) \rightarrow 0$;

(c) $\left\|\varphi_{n}-\varphi\right\| \rightarrow 0$.

Proof. First suppose that (a) holds, so that $\varphi_{n} \rightarrow \varphi$ in $M\left(H^{\infty}(D)\right)$. To prove that (b) holds, first note that by Corollary 9 , we can assume (by deleting a finite number of terms from the sequence $\left.\left(\varphi_{n}\right)_{n=1}^{\infty}\right)$ that

$$
\sup \left\{d_{D}\left(\varphi_{n}, \varphi\right): n \in \mathbf{Z}^{+}\right\}<1 .
$$

In particular, $\varphi_{n} \in G(\varphi)$ for each $n$. Thus for each $n$ there exists $w_{n} \in D$ such that $L_{\varphi}\left(w_{n}\right)=\varphi_{n}$. If $G(\varphi)=\{\varphi\}$, then $\varphi_{n}=\varphi$ for each $n$, and so (b) holds. Hence we can assume that $G(\varphi) \neq\{\varphi\}$. Thus

$$
\begin{aligned}
\left|w_{n}\right| & =d_{D}\left(w_{n}, 0\right) \\
& =d_{D}\left(L_{\varphi}\left(w_{n}\right), L_{\varphi}(0)\right) \\
& =d_{D}\left(\varphi_{n}, \varphi\right) \quad \text { for all } n \in \mathbf{Z}^{+},
\end{aligned}
$$

where the second equality above follows from equation (6.1) of [7]. By the above equality, to prove that (b) holds we need only show that $w_{n} \rightarrow 0$. Suppose that this fails. Then the above equality and inequality (14) show there exists a subsequence $\left(w_{n_{j}}\right)_{j=1}^{\infty}$ and $w \in D \backslash\{0\}$ such that $w_{n_{j}} \rightarrow w$ as $j \rightarrow \infty$. Because $L_{\varphi}$ is continuous, we have $\varphi_{n_{j}}=L_{\varphi}\left(w_{n_{j}}\right) \rightarrow L_{\varphi}(w)$ as $j \rightarrow \infty$. Recalling that $\varphi_{n} \rightarrow \varphi$, we conclude that $L_{\varphi}(w)=\varphi$, which implies (because $L_{\varphi}$ is injective) that $w=0$. This contradiction completes the proof that (a) implies (b).

Now suppose that (b) holds. To prove that (c) holds, note that by deleting a finite number of terms from the sequence $\left(\varphi_{n}\right)_{n=1}^{\infty}$, we can assume that $\varphi_{n} \in$ $G(\varphi)$ for all $n \in \mathbf{Z}^{+}$. As above, let $w_{n} \in D$ be such that $L_{\varphi}\left(w_{n}\right)=\varphi_{n}$. Once again, if $G(\varphi)=\{\varphi\}$ then $\varphi_{n}=\varphi$ and (c) obviously holds, so we can assume that $G(\varphi)=\{\varphi\}$. Thus by equation (15), we have $w_{n} \rightarrow 0$, and so we can assume that $\left|w_{n}\right|<\frac{1}{2}$ for all $n$. Let $f \in H^{\infty}(D)$. Then

$$
\begin{aligned}
\left|\varphi_{n}(f)-\varphi(f)\right| & =\left|\left(f \circ L_{\varphi}\right)\left(w_{n}\right)-\left(f \circ L_{\varphi}\right)(0)\right| \\
& \leq 2\left|w_{n}\right|\left\|f \circ L_{\varphi}\right\|_{\infty} \\
& \leq 2\left|w_{n}\right|\|f\|_{\infty},
\end{aligned}
$$

where the first inequality comes from the Cauchy integral formula. The above inequality implies that $\left\|\varphi_{n}-\varphi\right\| \rightarrow 0$, completing the proof that (b) implies (c).

Clearly (c) implies (a), completing the proof of the corollary. 


\section{ACKNOWLEDGMENT}

The second author thanks the Mathematics Department of the University of Bern, where part of this work was done, for its hospitality.

\section{REFERENCES}

1. Herbert S. Bear, Lectures on Gleason parts, Springer-Verlag Lecture Notes in Math., Vol . 121, 1970.

2. A. Dufresnoy, Sur les compacts d'interpolation du spectre de $H^{\infty}(D)$, Studia Math. 43 (1972), 235-244.

3. Theodore W. Gamelin, Uniform algebras, Prentice-Hall, Englewood Cliffs, NJ, 1969.

4. T. W. Gamelin and John Garnett, Distinguished homomorphisms and fiber algebras, Amer. J. Math. 92 (1970), 455-474.

5. John B. Garnett, Bounded analytic functions, Academic Press, New York, 1981.

6. Kenneth Hoffman, Banach spaces of analytic functions, Prentice-Hall, Englewood Cliffs, NJ, 1962.

7. __ Bounded analytic functions and Gleason parts, Ann. of Math. 86 (1967), 74-111.

Department of Mathematics, Michigan State University, East lansing, Michigan 48824

Department of Mathematics, Bucknell University, Lewisburg, Pennsylvania 17837 